\title{
Exponentially Growing Dark Matter
}

\section{A new model explains the current density of dark matter by proposing that conventional matter converted to dark matter in the early Universe.}

\author{
By Erika K. Carlson
}

C osmologists infer how much dark matter is in our Universe from observations of the cosmic microwave background (CMB). For dark matter theories to be viable, they must be able to reproduce this dark matter density. Now, Torsten Bringmann of the University of Oslo, Norway, and colleagues propose a new mechanism by which our Universe could have arrived at its present-day dark matter density [1].

Explanations for today's dark matter density generally involve either freeze-out or freeze-in models. In freeze-out models, a large initial quantity of dark matter is in equilibrium with the plasma of standard model particles in the early Universe. As the Universe expands and cools, processes that destroy dark matter particles outpace those that create it, until the dark matter density decreases to its present-day level. In freeze-in models, the Universe starts with little or no dark matter, and the plasma of standard model particles births dark matter until its density reaches today's levels.

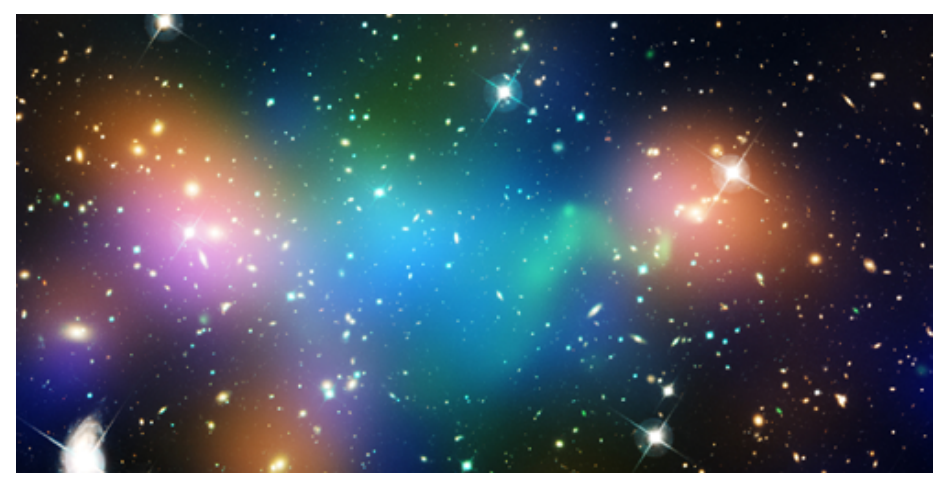

Credit: NASA, ESA, CFHT, CXO, M. J. Jee (University of California, Davis); A. Mahdavi (San Francisco State University).
Bringmann and colleagues propose another trajectory for dark matter's density. They suggest that a small initial quantity of dark matter in the early Universe could interact with standard model particles such that these particles transform into dark matter particles themselves. By converting standard model particles to the dark side-and allowing the new recruits to do the same to other standard model particles-dark matter could reproduce much more rapidly than in freeze-in models, growing exponentially in this dense early environment. The process naturally slows and then stops as the Universe expands, eventually reaching the dark matter density that we observe today. Such a history might have consequences for the CMB's power spectrum and other present-day properties of the Universe, the researchers say, meaning this potential mechanism could be supported or ruled out by future observations.

Erika K. Carlson is a Corresponding Editor for Physics based in New York City.

\section{REFERENCES}

1. T. Bringmann et al., "Dark matter from exponential growth," Phys. Rev. Lett. 127, 191802 (2021). 